\title{
Comparison of an NVG Model with Experiments to Elucidate Temporal Behaviour
}

\author{
Paul J. Thomas ${ }^{1}$, Robert S. Allison ${ }^{2}$, Sion Jennings ${ }^{3}$, Todd Macuda ${ }^{3}$, James Zacher ${ }^{2}$, Henok \\ Mehbratu $^{2}$, Richard Hornsey ${ }^{2}$ \\ ${ }^{1}$ Topaz Technology Inc., Toronto, Canada \\ ${ }^{2}$ York University, Department of Computer Science, Toronto, Canada \\ ${ }^{3}$ National Research Council of Canada, Institute for Aerospace Research, Ottawa, Canada
}

\begin{abstract}
Expected temporal effects in a night vision goggle (NVG) include the fluorescence time constant, charge depletion at high signal levels, the response time of the automatic gain control (AGC) and other internal modulations in the NVG. There is also the possibility of physical damage or other non-reversible effects in response to large transient signals. To study the temporal behaviour of an NVG, a parametric Matlab model has been created. Of particular interest in the present work was the variation of NVG gain, induced by its automatic gain control (AGC), after a short, intense pulse of light. To verify the model, the reduction of gain after a strong pulse was investigated experimentally using a simple technique. Preliminary laboratory measurements were performed using this technique. The experimental methodology is described, along with preliminary validation data.
\end{abstract}

Keywords: Night Vision Goggles, NVG, automatic gain control, AGC, modeling, temporal behaviour

\section{INTRODUCTION}

Night vision goggles (NVG) are widely used in civilian and military aviation. The models and types of NVGs available to the pilot have been increasing and there has been a desire to model NVG capability and to compare the performance of various types of NVGS. The investigating team was interested in improving the capability of an existing mathematical model ${ }^{1}$ of $\mathrm{NVG}$ performance to incorporate the transient response of the image intensifier tube to changing global scene illumination levels.

Aviation NVGs have embedded circuitry that will automatically adjust to changing light levels so that pilots can look from light to dark areas within a scene and still see useful detail. Normally, the response of an NVG would be influenced by the fluorescence time constant, charge depletion at high signal levels, the response time of the automatic gain control (AGC) and other internal modulations in the NVG. Very large transient light signals may cause physical damage or other non-reversible effects to the device. The damaging effects were not examined in the current effort which was limited to understanding NVG response to normal transitions from moonlight to starlight (or darker) conditions. While various temporal factors affect NVG response, as indicated in Table 1, the transient response is primarily driven by the automatic gain control circuitry (AGC) that is embedded in aviation NVGs. The AGC has the longest time constant of all the temporal factors excluding overload recovery. Since the primary interest was the AGC response, the modeling and experiments were designed to measure response times in the millisecond regime.

Table 1 Temporal Factors in an NVG

\begin{tabular}{|l|l|l|l|}
\hline Factor & Units & Nominal value & Notes \\
\hline Transit Time & nsec & $<100$ & Electron transit through NVG \\
\hline Charge Depletion Recovery & $\mu \mathrm{sec}$ & $<100$ & Microchannel plate recharging after an event \\
\hline Fluorescence Decay & $\mu \mathrm{sec}$ & 700 & See reference [2] \\
\hline AGC Response & $\mathrm{msec}$ & $10^{0}-10^{3}$ & Primary model parameter of interest \\
\hline Overload Recovery & $\mathrm{sec}$ & Unknown & Overload situation not observed \\
\hline
\end{tabular}

Head- and Helmet-Mounted Displays XII: Design and Applications 


\section{MODEL ELEMENTS}

The AGC model fits into an overall modeling process reported on earlier [1]. The overall modeling process builds an image sequence using the steps shown in Figure 1. Model inputs are images that represent the scene radiance distribution. These input images were read sequentially from a series of files. Each image was reformatted and transformed to provide an irradiance distribution at the input of the NVG. It was assumed that the spatial variation in the image corresponded to radiance variations in the scene that were appropriate for the spectral sensitivity of the NVG

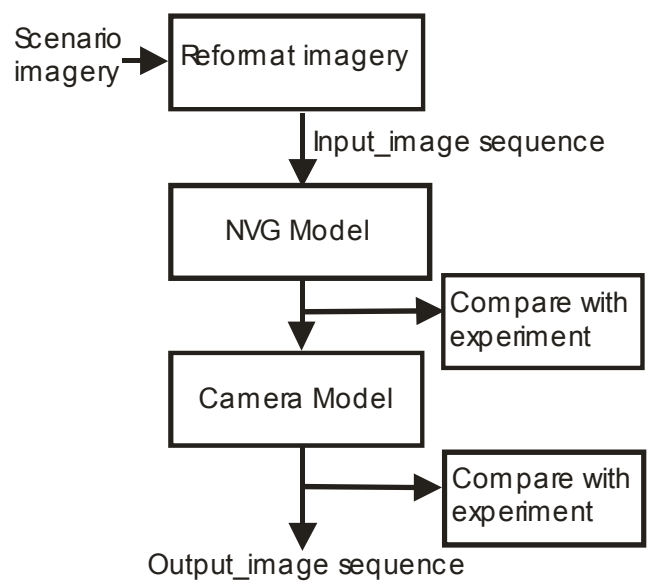

Figure 1 Elements of an NVG Model

Next, this optical irradiance distribution was converted to a radiance distribution at the output (i.e. the fluorescent screen) of the NVG[1]. The output image could be monitored for comparison with experiments with a human observer. (In such a comparison, the effects of the eyepiece and the human eye must be considered as well.)

The NVG model was based on a weak-signal algorithm. In a single iteration of the model (a 'time slice'), the duration of the time slice was selected so that the probability of a photoelectron at a given micro-channel of the MCP was much less than unity. Longer time durations/larger signals were modeled by repeated time slices and integrating the corresponding output images. Between iterations, the NVG parameters were changed as needed to model temporal behaviour. Stronger signals were treated by reducing the time associated with each of the iterations, thus maintaining the weak-signal condition. Of course, additional iterations were then required for a constant total integration time of the image. Characteristics of the integrated image, such as mean signal, were calculated after each iteration.

\subsection{AGC Model}

The details of the implementation of the AGC function are not generally disclosed by the manufacturer, therefore, the AGC was treated as a black box. The model does not account for the precise location or implementation of the gain control. There are many possible methods of implementing an AGC, such as gating the photocathode proximity focusing voltage or controlling the microchannel plate current or voltage. Each method of implementation may affect other aspects of the NVG image such as halo and channel noise. In this effort, automatic gain control was implemented by changing the MCP voltage. Other methods of controlling the gain such as duty cycle modulation could be inserted into the model. Because of the iterative nature of the NVG image creation, as described above, temporal variations of parameter values were readily incorporated with realistic time constants.

As outlined above, the automatic gain control (AGC) function was implemented by calculating image characteristics during each iteration of the NVG core model. The average signal in the image (over a suitable integration time) determined the gain level which was used to modify the model parameters. A block schematic of this process is shown in Figure 2. 


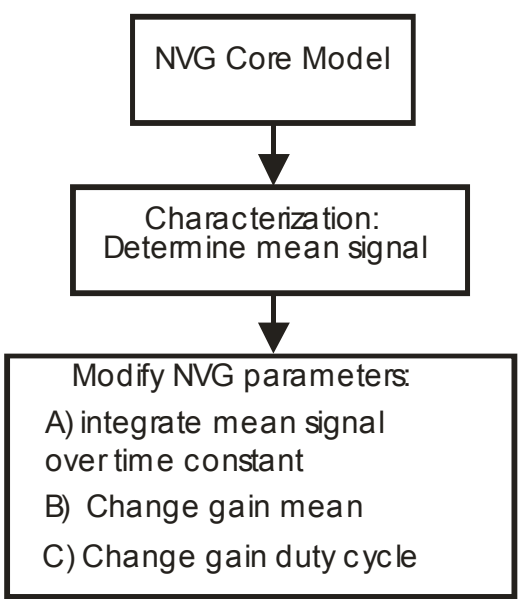

Figure 2 Block Schematic of AGC Model

\section{EXPERIMENTAL DETERMINATION OF PULSED LASER RESPONSE}

To gather the parametric data, an apparatus was prepared as shown in Figure 3. Two LEDs were used to illuminate a large-area white screen that served as the scene background. The first LED served as a continuous background light source while the second LED could be pulsed to provide additional background illumination. The intent of the background light sources was to establish a defined level of ambient illumination and fix the state of the automatic gain control (AGC) of the NVG. The stability of the intensity of the background light was monitored with a photodiode/picoammeter that was occasionally inserted into the optical path.

In the center of the screen a hole was cut. A mirror was placed behind this aperture and a short pulse from a gated semiconductor laser was reflected though the hole and along the optical axis of the NVG. The laser pulse either acted as a probe of the current NVG state or, if sufficiently intense, further changed the NVG state. The hole was large compared to the angular size of the laser source, so that the regions of the NVG illuminated by the laser spot and the background light could be readily separated during image processing. The time durations of the laser pulse and the light source were selected to facilitate the measurement of selected temporal factors of the NVG. The eyepiece lens of the NVG was removed and the output on the fluorescent screen of the NVG was imaged by a CMOS camera.

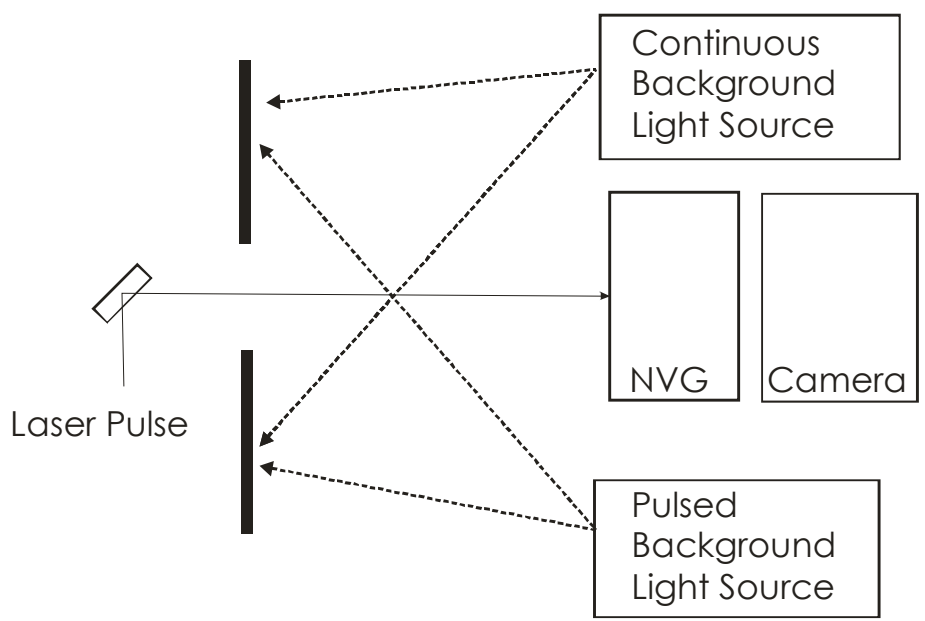

Figure 3 Short-Pulse Laser Experimental Scenario

The observed NVG signal in response to a laser pulse was affected by the state of the AGC. The laser pulse was used in this case as a 'probe' of the NVG gain. If the laser pulse energy is constant, the signal level of the image of the laser 
pulse should also be constant. Variations in the signal strength of the image of the laser pulse were attributed to changes in the NVG gain.

The NVG was operated normally, except that the eyepiece on one side was removed and replaced by a CMOS imager. A representative camera image (see Figure 4) shows two optical distributions - a localized event from the point source and a broad feature due to the background light. The background light is the image of a broad circular distribution of light on a white screen. Although the background appears black in Figure 4, the white screen covered the field of view of the image, and shows up only where illuminated by the background light. Behind a hole in the white screen was the laser source. When imaged by the NVG, the laser source had a bright central region, which was used in the measurements, and a fainter halo that was ignored.
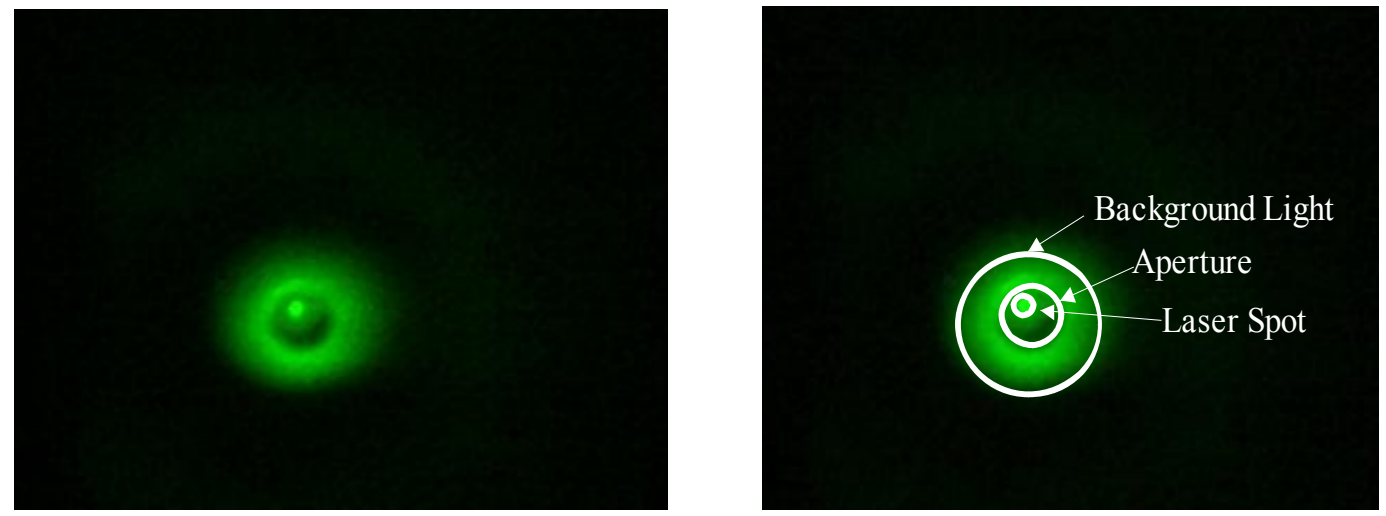

Figure 4 Representative Camera Image

Although the background image was white and the laser was red, a monochrome green image is produced by the NVG. This green light showed up in the green, blue and red channels of the colour camera, although the red and blue signals were greatly attenuated with respect to the green channel. In the analysis of the AGC behaviour, the green channel alone was used for quantitative results, while all colours may be present in the photographs and imagery or used to clarify effects of camera saturation.

\subsection{Setup and Timing in the Experiment}

The control and timing of the data gathering system is shown in Figure 5, which gives the control/timing signals for the two light sources, the variable time delay and the camera integration. The accuracy of the time delay of the laser pulse, $t_{\text {delay }}$, determined the accuracy of the measurement of AGC response. The background light source was turned on for $800 \mathrm{~ms}$. The resulting scene illumination was estimated to be in a moonlight region. When the background source was turned off - resulting in no scene illumination and maximum NVG gain - a variable time delay was triggered. After the variable time delay, the laser and camera were turned on simultaneously. At this point, the camera captured an image of the laser pulse. The resulting intensity of the laser pulse was used to determine the NVG gain. The process was then repeated with different delay times.

The timing signals are summarized Table 2. The period of the master clock was selected to ensure that the AGC of the NVG was in a stable state corresponding to the power in the background-light signal. The timing signals were monitored with the aid of a $60 \mathrm{MHz}$ digital oscilloscope, which also provided the estimates of timing uncertainty.

The laser was a semiconductor device. The pulse duration was manually adjusted to prevent the signal level of the laser pulse on the camera from saturating, while the delay time was varied manually to monitor the gain state of the NVG at different times after the shut-off of the (relatively strong and delocalized) background signal. The delayed signals from this master clock had a timing accuracy of about $1 \mu \mathrm{sec}$ and a waveform risetime (10 to $90 \%$ points) that was better than $0.05 \mu$ sec. Amplitude stability was better than $2 \%$ and amplitude overshoot was about $5 \%$ with a frequency spectrum consistent with the risetime. This level of timing and amplitude stability ensured that uncertainties in timing were not an experimental limitation.

The laser source showed no significant waveform distortions at pulse durations of $300 \mathrm{nsec}$ or above. Although some degradation of the LED pulse waveform occurred due to the intrinsic capacitance of the LED and the $50 \mathrm{ohm}$ impedance of the voltage source, the fall time of the LED optical waveform (10 to 90\%) was less than $2 \mu$ sec. This fall time is not 
an intrinsic limitation of the method, and was at least an order of magnitude shorter than the expected time constants of the NVG of interest to this work. A small amount of spontaneous emission from the laser was present even when the laser was turned off. The effect of this 'leakage' light was reduced by increasing the duty cycle of the pulsed laser and by measuring the DC component of the pulse. The DC component was later removed from the imagery as needed by background subtraction or other image processing.

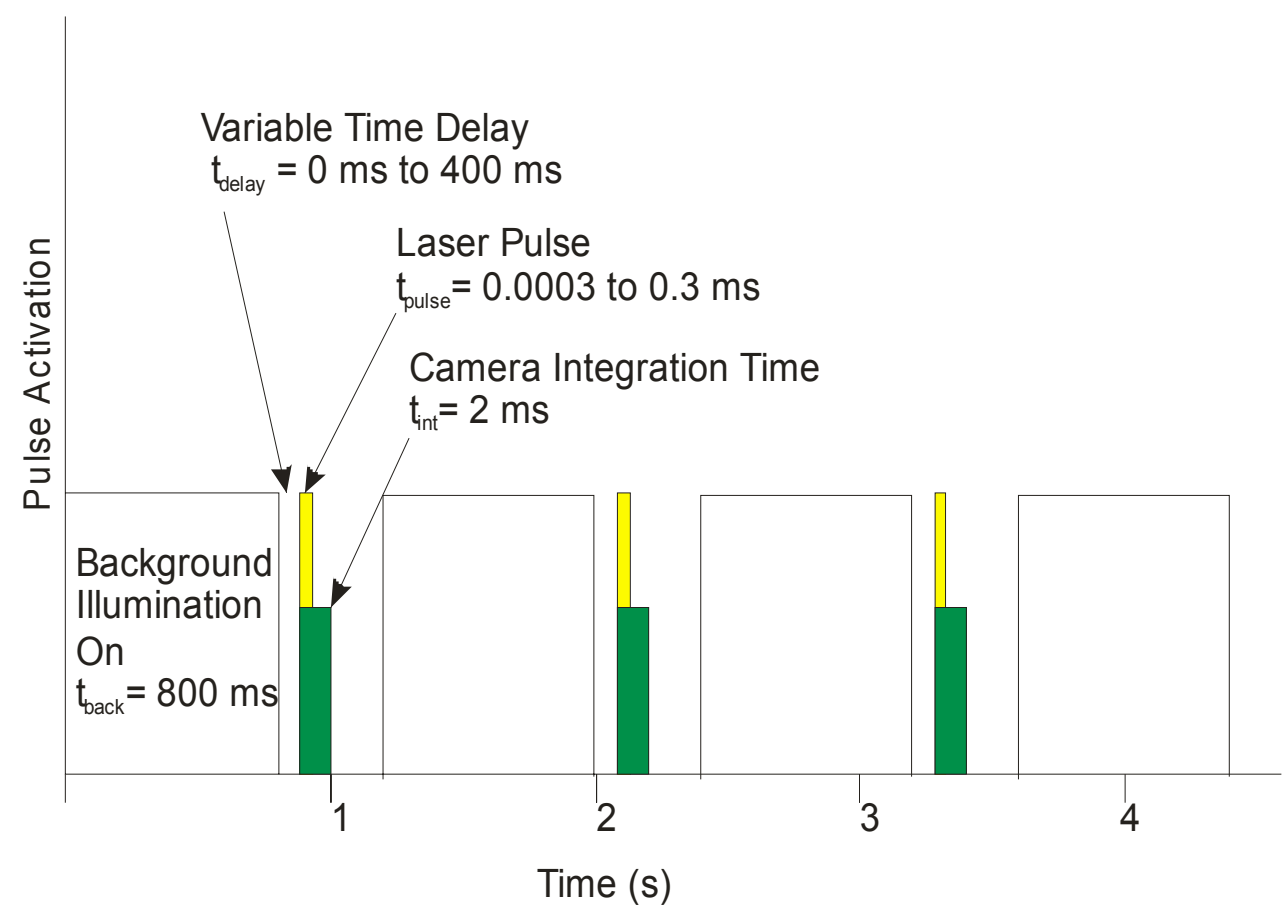

Figure 5 Experimental Control Signals

The delay time of the laser pulse could be controlled from $0 \mu \mathrm{sec}$ to about $10^{6} \mu \mathrm{sec}$ with an uncertainty of $\pm 1 \mu \mathrm{sec}$. This level of precision in the delay time was expected to be sufficient for the temporal behaviour patterns of interest. The camera had external-triggering capability that was triggered on the start of the laser pulse.

Table 2 Experimental Timing

\begin{tabular}{|l|l|}
\hline Signal & Time (ms) \\
\hline Master clock (period) & 1200 \\
\hline Background illumination on & 800 \\
\hline Variable time delay & 0 to 400 \\
\hline Laser Pulse & 0.0003 to 0.3 \\
\hline Camera Integration Time & 2 \\
\hline
\end{tabular}

A search was conducted for temporal modulations of the NVG gain, by the acquisition of a large number of images of the laser spot incident on the NVG, with the background light held at a constant illumination level. If the NVG gain were modulated, this modulation should appear as with a corresponding modulation of the intensity of the image of the laser spot. The duration of the laser spot is a constraint on this method. It is possible to have an accidental synchronicity between the NVG modulation and the laser pulse repetition rate. This factor was eliminated by the use of several laser repetition rates. If the NVG modulation period is shorter than the duration of the laser pulse, a modulation on the laser spot intensity may not be present. For these experiments, a laser pulse of $500 \mu \mathrm{sec}$ duration was used, so that internal 
modulations of the NVG up to $2 \mathrm{kHz}$ should affect the experiment. Preliminary measurements on a single test NVG did not uncover any modulation in the above frequency range.

\subsection{Image Processing}

The objective of the image processing was to determine the signal strength in the laser spot. Image processing routines (see Table 3) were created in Matlab to extract the laser spot signal. First the spot was isolated from the wide area background. From the perspective of image processing, the laser spot was identified by finding its centroid and size, and then the energy within the spot was calculated by integrating over only that region.

Table 3 Image Processing Routines

\begin{tabular}{|l|l|}
\hline Processing Step & Notes \\
\hline $\begin{array}{l}\text { Region of interest } \\
\text { (ROI)identification }\end{array}$ & $\begin{array}{l}\text { This routine looks for the laser spot, which was the region of maximum energy } \\
\text { ("brightness") in the image. As the background illumination was off at this point, the } \\
\text { only bright spot in the image was the laser. Draw a box around the maximum that is } \\
\text { not much bigger than the spot. }\end{array}$ \\
\hline Centroid of laser spot & $\begin{array}{l}\text { Based on the above ROI, a small region was defined, which was expected to contain } \\
\text { only the laser spot. The row and column centroid positions in this region were } \\
\text { determined by a centre-of-mass algorithm. }\end{array}$ \\
\hline Background signal strength & $\begin{array}{l}\text { Not used for the results reported here. However when looking at AGC changes that do } \\
\text { not end at maximum sensitivity, this feature could be used. The dark background was } \\
\text { found to be stable to better than 1\%, and did not require frequent monitoring. }\end{array}$ \\
\hline Laser spot size & $\begin{array}{l}\text { Draw a threshold at 5\% of maximum and then integrate the signal within the box to } \\
\text { arrive at an "energy" of the laser spot. This routine proved to be robust against } \\
\text { threshold changes (2\% to 10\%) and box size changes. }\end{array}$ \\
\hline Laser spot background & $\begin{array}{l}\text { To remove the dc component of the laser light, the laser background was determined } \\
\text { in a separate experiment (by disabling the laser-enable). This background distribution } \\
\text { was then subtracted from the laser spot image. }\end{array}$ \\
\hline Laser spot integrated signal & $\begin{array}{l}\text { The laser spot integrated signal summed the signals in a region about the centroid } \\
\text { determined by the laser spot size. }\end{array}$ \\
\hline Camera noise & $\begin{array}{l}\text { Camera 'noise' (including stray room light) was determined in a separate experiment, } \\
\text { with no light sources enabled. The magnitude of this camera noise was small enough } \\
\text { to be ignored. }\end{array}$ \\
\hline
\end{tabular}

However, there were other sources of noise and signal that needed to be eliminated from the final calculations. For example, the spontaneous-emission from the laser created a background signal that needed to be extracted from the signal. This background signal beneath the laser spot was estimated from the local neighbourhood in a separate experiment with the laser pulse disabled, and then subtracted from the laser pulse energy. The contribution of camera noise to the signal was minimized by the selection of a small region of interest, a short camera integration time and an intense laser pulse. The measurements were performed in a light-tight enclosure in a darkened room to reduce stray room light.

\subsection{Sources of Error}

The goal of the modeling effort described here was to examine the temporal response of the NVG AGC. Using the procedure outlined above, the accuracy of the measurement of the NVG response depended on the timing accuracy, the uncertainty in the relative gain and modeling errors. The major factors that contributed to this uncertainty are outlined in Table 4. There were additional environmental and other factors that were not considered in this modeling effort but may have an effect on the NVG. 
Fluctuations in the laser pulse energy were measured by replacing the NVG and camera of Figure 1 with a photodiode. The fluctuations in the pulse amplitude and shape were then monitored with an oscilloscope. As expected, these fluctuations were small because the laser was powered with a precision DC voltage source and the temperature and other (laboratory) environmental parameters were approximately constant.

Camera noise was measured by acquiring a number of images of the laser spot, with the camera but without the NVG. The integrated signal in a region of interest (ROI) around the spot centre was then measured for each image. The fluctuation in the measured signal was used as an estimate of camera noise. Stray light and image saturation were avoided by experiment design.

Table 4 Contributions to Variations in Laser Spot Signal

\begin{tabular}{|l|l|}
\hline Factor & $\begin{array}{l}\text { Estimated } \\
\text { Magnitude (\%) }\end{array}$ \\
\hline Measured fluctuations of the laser pulse energy & 2 \\
\hline Camera noise & $<2$ \\
\hline Background light & $<1$ \\
\hline NVG noise & $<2$ \\
\hline NVG gain fluctuations/drift & $<2$ \\
\hline Image processing errors & $<1$ \\
\hline Temporal modulation of NVG gain & 0 \\
\hline $\begin{array}{l}\text { Total uncertainty } \\
\text { Assuming independent errors, combined in quadrature }\end{array}$ & $<4$ \\
\hline
\end{tabular}

Error from the background light was reduced by constraining the region of interest used for calculation of the laser-spot signal to include a small region around the spot. Background light was measured in image frames that did not contain the laser spot, and the mean background was subtracted from the laser spot. The residual uncertainty was estimated from a qualitative examination of images. NVG noise and gain fluctuations were not explicitly measured here, but were estimated from the NVG model. Image processing errors can arise from anomalous signals, a finite region of interest, truncation errors in computations and other factors. Such errors were estimated from the corresponding effect on synthetic imagery.

As discussed previously, temporal modulation of the gain of the NVG can reduce the signal from a (periodic) short laser pulse, if the period of the gain modulation is not in phase with that of the laser pulses. This modulation behaviour was not exhibited by the NVG used for experimental validation of the model.

\section{PRELIMINARY MEASUREMENTS}

Preliminary measurements from a typical third generation NVG system - obtained using the methods described above are shown in Figure 6. The background light was turned off at $\mathrm{t}=0$. The 'relative gain' (ordinate axis) was determined, as described in the image processing section above, by integrating the signal in the laser spot. This signal-integral has been normalized so that the high-gain state has a value of 100 .

In Figure 6, five images were acquired at each time delay. The spread in the spot-energy measurements for a fixed time delay is consistent with the uncertainties identified in Table 4. Within the experimental uncertainty, the AGC response appears to be linear until close to the endpoint. The full-scale response is approximatel $60 \mathrm{~ms}$.

This NVG response to a step change in input light level is, to first order, better modeled by high order response dynamics rather than a proportional control algorithm. Exact modeling of the system control is beyond the scope of this paper. However, the precise value of the response time is sensitive to the control algorithm assumed. 


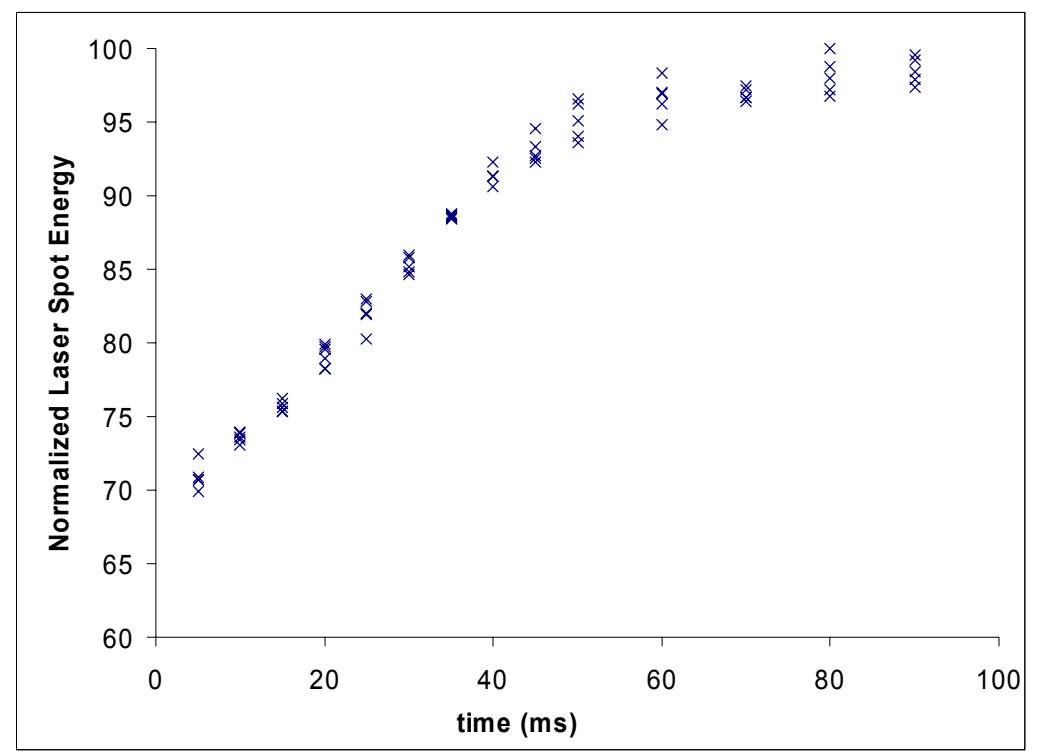

Figure 6 Relative Gain Versus time delay

CONCLUSIONS

The automatic gain control in aviation NVGs enables pilots to look from brightly illuminated areas to dimly illuminated areas. The AGC helps to maintain an acceptable level of image contrast in both situations. With the introduction of many new NVG models into the marketplace, there is a desire to compare their performance. This paper outlines a simple method for characterizing the response of the NVGs to large variations in light level. The results of the characterization can be imported into NVG modeling software or used for comparative evaluations. Preliminary results using this characterization technique show a full scale response to a change in light levels within $60 \mathrm{~ms}$.

\section{REFERENCES}

1. Paul J. Thomas, R.S. Allison, T. Macuda, S. Jennings, K. Yip, E. Savchenko, I. Tsang, R. Hornsey, "Experimental validation of synthetic imagery for night vision goggles", SPIE, Vol. 5442, paper 03, April 2004 .

2. W.E. Flynt, "Characterization of Some Common CRT Phosphors", in "Ultrahigh Speed and High-Speed Photography”, Photonics and Videography '89, G.L. Stradling, editor, Proc SPIE Vol 1155, pp 123-130, 1989. 